\title{
Akademische multizentrische Studien im Fokus: Auswirkungen der Arzneimittelgesetzgebung auf Therapieoptimierungsstudien in der Hämatologie/ Onkologie
}

\author{
Kristina Ihrig ${ }^{\mathrm{a}}$ Birgit Fath $^{\mathrm{b}} \quad$ Michael Fuchs $^{\mathrm{c}} \quad$ Michael Hallek $^{\mathrm{b}} \quad$ Norbert Marschner $^{\mathrm{d}}$ \\ Ralph Naumann ${ }^{\mathrm{e}}$ Christoph Röllig $^{f}$ Susanne Saußele $^{\mathrm{g}}$ Hans Tesch $^{\mathrm{h}}$ Nicola Gökbuget ${ }^{\mathrm{e}, \mathrm{i}}$ \\ ${ }^{a}$ Kompetenznetz Leukämien, Medizinische Klinik II, Hämatologie und Onkologie, Studienzentrale, Klinikum der Goethe-Universität, \\ Frankfurt a.M., \\ ${ }^{\mathrm{b}}$ Kompetenznetz Maligne Lymphome e.V., Universitätsklinikum Köln AöR, \\ ${ }^{c}$ Deutsche Hodgkin-Studiengruppe, Studienzentrale der GHSG, Medizinische Klinik I, Klinikum der Universität zu Köln, Köln, \\ ${ }^{\mathrm{d}}$ Onkologische Schwerpunktpraxis, Freiburg i.Br., \\ ${ }^{e}$ Arbeitskreis Arzneimittelgesetz der Deutschen Gesellschaft für Hämatologie und Medizinische Onkologie (DGHO e.V.), \\ Stiftungsklinikum Mittelrhein, Koblenz, \\ ${ }^{f}$ Studienallianz Leukämien und Kompetenznetz Leukämien, Medizinische Klinik und Poliklinik I, \\ Universitätsklinikum der Technischen Universität Dresden, Bereich Klinische Studien, SAL-Studienzentrale, Dresden, \\ ${ }^{9}$ Deutsche Studiengruppe für die chronische myeloische Leukämie und Kompetenznetz Leukämien, III. Medizinische Klinik, \\ Hämatologie/Onkologie, Universitätsmedizin Mannheim, Mannheim,

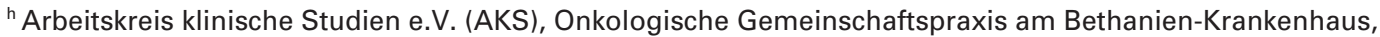 \\ Deutsche Studiengruppe für akute lymphatische Leukämie des Erwachsenen, Kompetenznetz Leukämien, Arbeitskreis Arzneimittelgesetz \\ der Deutschen Gesellschaft für Hämatologie und Onkologie (DGHO e.V.), Medizinische Klinik II, Hämatologie und Onkologie, Klinikum der \\ Goethe-Universität, Frankfurt a.M., Deutschland
}

\section{Schlüsselwörter}

IIT · Investigator-initiierte Studien · 12. AMG-Novelle .

TOS · Therapieoptimierungsstudien

\section{Zusammenfassung}

Hintergrund: Zweck dieser Erhebung war die Erfassung der Auswirkungen gesetzlicher Rahmenbedingungen für klinische Studien auf Prüfzentren hämatologisch-onkologischer Investigator-initiierter Studien (IIT). Methode: Fragebögen wurden an Leitungen hämatologisch-onkologischer Prüfzentren versandt. Ergebnisse: Teilnehmende Zentren an IIT weisen eine gute Infrastruktur und umfassende Studienerfahrung auf. Je nach Erkrankung wird ein hoher Anteil der Patienten in Studien behandelt, um Therapieergebnisse zu verbessern. Dennoch werden 35\% der Umfrageteilnehmer ihre Teilnahme an IIT reduzieren, da unter anderem für die aufwendige Organisation keine ausreichende Finanzierung für Studienpersonal besteht. Schlussfolgerungen: Die Studienlandschaft der Hämatologie/Onkologie mit ihren akademischen Multicenterstudien ist gefährdet. Dies hat weitreichende Folgen für Patienten, da qualitativ hochwertige Studien an vielen Standorten nicht aktiviert werden und so wertvolle Daten für die Optimierung der Therapien verloren gehen. Um diese Entwicklung zu stoppen, ist eine Änderung der Rahmenbedingungen durch Gesetzgeber, Überwachungsbehörden und Kostenträger erforderlich.
Keywords

IIT · Investigator-initiated trials · German drug law .

TOS · Therapy optimization trials

\section{Summary}

Focus on Academic Multicenter Trials: Impact of the German Drug Law on Hematological/Oncological Therapy Optimization Trials

Background: The purpose of this study was to evaluate the effect of legal regulations for clinical trials on study centers participating in investigator-initiated trials (IITs) in the field of hematology/oncology. Method: Questionnaires were sent out to the heads of hematology-oncology study centers. Results: Medical units participating in IITs have a good infrastructure and extensive experience in clinical trials. Depending on indication, a high proportion of patients have been treated in studies with the purpose to improve outcome. However, $35 \%$ of the responders will reduce their participation in IITs in the future due to a lack of financial support for staff involved in the extensive organizational tasks. Conclusions: The widely recognized research field in therapy optimization trials in hematology and oncology in Germany is at risk. This will have negative effects on the patients as highly sophisticated protocols will no longer be initiated in several study centers, resulting in the loss of valuable data for the improvement of patient therapy and outcome. To stop this development, legislators as well as regulatory authorities and health insurances need to make the necessary changes in the legal framework.

\section{KARGER \\ Fax +497614520714 \\ Information@Karger.com}

www.karger.com (c) 2013 S. Karger GmbH, Freiburg

0378-584X/13/3614-0023\$38.00/0

Accessible online at:

www.karger.com/onk
Dr. med. Nicola Gökbuget

Medizinische Klinik II, Hämatologie und Onkologie

Klinikum der Goethe-Universität

Theodor-Stern-Kai 7, 60590 Frankfurt, Deutschland

goekbuget@em.uni-frankfurt.de 


\section{Einleitung}

Investigator-initiierte Studien (IIT) und insbesondere Therapieoptimierungsstudien (TOS) besitzen in der Hämatologie/ Onkologie beginnend mit der Gründung von Multicenterstudiengruppen im Rahmen einer Initialförderung durch das Bundesministerium für Bildung und Forschung (BMBF) im Jahre 1978 eine langjährige Tradition. Weitere positive Impulse wurden im Jahr 1999 durch das BMBF als Förderer krankheitsbezogener Kompetenznetze gesetzt, wie z.B. die Förderung der Kompetenznetze akute und chronische Leukämien (KNL) sowie maligne Lymphome (KML). Im Mittelpunkt steht die Behandlung von Patienten im Rahmen von Studien, die jedoch nicht in erster Linie der Zulassung einer Prüfmedikation dienen. Diese akademischen multizentrischen Studien zielen vor allem auf die Optimierung multimodaler Therapiekonzepte bei seltenen Erkrankungen ab, die neben Kombinationstherapien mit verschiedenen zugelassenen Medikamenten auch eine Strahlentherapie oder Stammzelltransplantation umfassen können.

Hämatologische Neoplasien sind selten und zählen größtenteils zu den Orphan Diseases (Definition in der Europäischen Union (EU): Inzidenz <5/10 000). Die besonderen Herausforderungen für IIT im Bereich der Hämatologie liegen daher in der Beteiligung einer großen Zahl medizinischer Einrichtungen (mehrere 100), mit dem Ziel, deutschlandweit und möglichst versorgungsnah Therapien anzubieten. Diese TOS verbinden Versorgung und Forschung miteinander. Den Studiengruppen im Bereich der Hämatologie ist dies in den letzten Jahren hervorragend gelungen und die Heilungschancen für Leukämien und Lymphome konnten substanziell verbessert werden. Gleichzeitig haben ihre wissenschaftlichen Aktivitäten auch international höchste Anerkennung gefunden [1].

Im Zuge der Harmonisierung nationaler Gesetze zur Studiendurchführung durch die europäische Clinical Trial(CT)Direktive wurden in Deutschland mit der 12. Arzneimittelgesetz(AMG)-Novelle 2004 die Rahmenbedingungen für IIT umfassend geändert: Akademische IIT müssen nun die gleichen Bedingungen wie Zulassungsstudien der pharmazeutischen Industrie erfüllen. Zugleich stehen den akademischen Sponsoren, die nicht markt-, sondern forschungsorientiert Studien veranlassen, keine ausreichenden finanziellen Mittel zur Verfügung, um die neuen und kostenintensiven Aufgaben zu bewältigen. Der Schwerpunkt der Probleme liegt in den sehr zeitaufwendigen Prozessen der Studienaktivierung besonders vieler Zentren, insbesondere in der Beantragung von Ethikvoten, der Vertragslogistik, der Wahrnehmung der Prüfarztpflichten bei Studien in der Versorgungssituation sowie beim Monitoring und in der Überwachung der Studien. Viele Probleme betreffen den akademischen Sponsor, aber auch teilnehmende Kliniken sind durch den hohen Verwaltungsaufwand stark belastet. In Deutschland und den Nachbarländern wird seither von den negativen Effekten dieser Änderung auf die unabhängige, versorgungsnahe Forschung berichtet [2-7]. Die Erfahrungen seit der Novelle zeigten, dass die Fortführung unabhängiger Studien zur Therapieoptimierung ernsthaft gefährdet ist. Um die Situation, die Motivation und Problemschwerpunkte für diese Studien in Deutschland näher zu charakterisieren und erstmals konkrete Zahlen zu erfassen, wurde im Rahmen des KNL eine Umfrage initiiert und in Kooperation mit anderen Netzen, Fachgesellschaften und Verbänden realisiert.

\section{Methodik}

Zielgruppe der Befragung waren die ärztlichen Leitungen deutscher hämatologisch-onkologischer Kliniken und Praxen, da diese Institutionen sowohl Teilnehmer als auch Initiatoren akademischer Multicenterstudien sind. In Kooperation mit den beteiligten Projektpartnern wurde ein Fragenkatalog entwickelt, der insgesamt 9 Themen mit 205 Items umfasste (Tab. 1). Der Fragebogen wurde per E-Mail über die Verteiler der Kooperationspartner an die Zielgruppe versendet.

Für den Initiator einer multizentrischen IIT ist die Darstellung der Qualifikation aller Prüfzentren und Prüfer für eine spezifische Studie eine neue, kosten- und zeitintensive Herausforderung. Um das Qualifikationsprofil der Zielgruppe beschreiben zu können, erfasste ein Fragenkomplex

Tab. 1. Fragenkatalog

Fragenabschnitt
Demografische Kennzahlen
Anforderungen der Ethikkommissionen an die Prüfzentren
Bedeutung von TOS

Problemschwerpunkte bei TOS

Modelle zur Unterstützung von Studienkliniken

Studienorganisation in Kliniken/Praxen Qualitätsmanagement in Kliniken/Praxen
Fragestellungen (Auswahl)

eigene Funktion, Art des Hauses, Anzahl Patienten, Studienbeteiligung

Infrastruktur Prüfzentrum

Rekrutierungsleistung, analoge Behandlung,

künftige Teilnahme, Motivation

Beantragung, Inspektionen, Apotheke, Abrechnung, Monitoring, Ausstattung

Reisedokumentare, Abrechnungsmodelle, Qualitätsmanagement, Datenbanken zur Reduktion der Verwaltung

Ausstattung

SOP, Zertifizierung

TOS $=$ Therapieoptimierungsstudien; $\mathrm{SOP}=$ Standard Operating Procedures. 
Daten zur Darstellung der bisherigen Teilnahme an indikationsbezogenen IIT mit und ohne Leitungsfunktion und Daten zur Darstellung der Eignung durch das Vorhandensein einer entsprechenden Infrastruktur, wie z.B. die Anbindung an notwendige Einrichtungen (Intensivstation, Labor Notfallversorgung usw.). Darüber hinaus wurde ganz generell die Breite der von den Ethikkommissionen geforderten Fortbildung zur Good Clinical Practice (GCP) in den Prüfzentren erhoben.

Weiterhin wurde, bezogen auf die einzelnen Indikationen, der Status der Rekrutierung für IIT als Schätzwert erfasst. Wichtig war hier die Frage nach der Motivation, Patienten in IIT einzubringen, aber auch die Abschätzung der künftigen Beteiligung an Studien und die Bedeutung studienanaloger Behandlungen. Um Prüfzentren zukünftig gezielt unterstützen zu können, wurden Kennzahlen zur Aktivierung von Studien erhoben und mögliche Problemfelder und die Wertigkeit von Unterstützungsmodellen evaluiert. Die Umfrage erfasste weiterhin die personelle Ausstattung von Prüfzentren in den Kliniken oder Praxen, aber auch das Qualitätsmanagement ganz generell bis hin zu Erfahrungen mit Audits und Inspektionen.

Die Daten wurden überwiegend deskriptiv ausgewertet; univariate statistische Vergleiche wurden mit dem $\chi^{2}$-Test durchgeführt (SAS 8.2).

\section{Ergebnisse}

\section{Teilnehmer}

Der Fragebogen wurde an die Leitungen von 134 Kliniken und 76 Praxen versandt. 66 Fragebögen konnten für die Auswertung berücksichtigt werden. Von den Teilnehmern waren 29 (44\%) hämatologischen Fachabteilungen von Universitätskliniken zugeordnet, 21 (32\%) anderen Kliniken und 16 ( $24 \%$ ) hämatologisch-onkologischen Praxen.

Alle beteiligten Zentren waren im Sinne einer hämatologischen Spezialabteilung/Praxis vollständig ausgerüstet und verfügten über eine Notfallversorgung. Die Umfrageteilnehmer behandelten geschätzt mindestens 4500 neu diagnostizierte Leukämie- und Lymphompatienten pro Jahr, und 95\% der Zentren waren mit mehr als 19 Betten bzw. Tagestherapieplätzen ausgestattet.

$80 \%$ der Zentren haben sich in den letzten 5 Jahren an mehr als 10 Studien beteiligt. Eine Leitungsfunktion nach der 12. AMG-Novelle wurde in $78 \%$ der teilnehmenden Institutionen bereits übernommen und eine mehr als 2-jährige Studienerfahrung lag in $95 \%$ der Zentren vor. Sie waren in der Regel mit einer Studienzentrale (oder äquivalenten Strukturen) ausgestattet (92\%), wobei die personelle Ausstattung variierte: Der Mitarbeiterstamm lag im Mittel für Prüfärzte mit ausschließlicher Tätigkeit als Studienarzt bei 0,4 (0-3), für Studienärzte mit zusätzlicher Tätigkeit in der Versorgung bei 3,3 (0-15) und für anderes Studienpersonal bei 3,8 Mitarbeitern (0-23).

Qualitätsmanagement in der Studienorganisation war in mehr als der Hälfte der Studienzentralen etabliert. 50\% ( $\mathrm{N}=$ 33) arbeiteten nach schriftlich festgelegten Verfahrensanweisungen (Standard Operating Procedures (SOP)) (62\% der Universitätskliniken, 35\% anderer Kliniken/Praxen). 28\% verfügten über ein zertifiziertes Qualitätsmanagement oder planten eine Zertifizierung. Zertifizierer bzw. Auditierer waren, geordnet nach Häufigkeit, die Deutsche Gesellschaft für Hämatologie und Medizinische Onkologie e.V (DGHO)/ Gesellschaft für Medizinische Innovation - Hämatologie und Onkologie mbH (GMIHO), die Kooperation für Transparenz und Qualität im Gesundheitswesen mbH (KTQ), der Deutsche Kraftfahrzeug-Überwachungs-Verein (DEKRA) und der Technische Überwachungsverein (TÜV).

\section{Bisherige Teilnahme an IIT}

IIT wurden für die Mehrzahl der malignen hämatologischen Erkrankungen durchgeführt; 59 (95\%) der Antwortenden hatten bereits an Studien von Leukämie- oder Lymphomstudiengruppen teilgenommen. In über der Hälfte der Zentren wurde geschätzt, dass ein hoher Anteil (>80\%) der Patienten mit akuter lymphatischer Leukämie (ALL), akuter myeloischer Leukämie (AML) oder Hodgkin-Lymphomen in IIT eingeschlossen wurde. Die Hälfte der Zentren schätzte, dass 50-80\% der Patienten mit chronischer lymphatischer Leukämie (CLL), chronischer myeloischer Leukämie (CML) oder hoch- bzw. niedrigmalignem Non-Hodgkin-Lymphom in IIT eingebracht worden waren. Mindestens jeder 2. Patient mit multiplem Myelom wurde in etwa 36\% der Prüfzentren in eine IIT eingeschlossen, und in etwa 90\% der Zentren wurde geschätzt, dass der Anteil der Studienrekrutierung für Patienten mit myelodysplastischem Syndrom (MDS) oder chronischen myeloproliferativen Erkrankungen (CMPE) unter 50\% lag.

\section{Probleme in der Durchführung von IIT}

Als wichtigste Probleme wurden der interne Organisationsaufwand im Verhältnis zu der geringen Fallzahl (75\% der Umfrageteilnehmer), die fehlende Stellenfinanzierung (66\%), ein hoher allgemeiner Verwaltungsaufwand (60\%), die geforderte Einhaltung der Dokumentationszeitpunkte (55\%) und die unzureichende Personaldecke aufgrund des Stellenmarktes $(33 \%)$ empfunden. Der Archivierungsaufwand oder drohende Inspektionen wurden ebenfalls als Problempunkte (39\% bzw. 32\%) thematisiert. Demgegenüber wurde die Akzeptanz von IIT intern bei Ärzten und Studienpersonal sowie Patienten von den Umfrageteilnehmern mit 80\%, 91\% und $86 \%$ als hoch eingeschätzt.

\section{Künftige Teilnahme an IIT}

Von den Umfrageteilnehmern stellten 35\% fest, dass sie ihre Teilnahme an prospektiven Leukämie- oder Lymphomstudien reduzieren wollten. Diese Angaben unterschieden sich nach Art der teilnehmenden Institution. 75\% der Praxen (12/16), 17\% der Universitätskliniken (5/29) und 25\% der anderen Kliniken (5/21) wollten künftig weniger oder gar nicht an IIT teilnehmen. Ein signifikanter Zusammenhang zwischen Indikation oder Fallzahl der Klinik und künftiger Rekrutierung konnte nicht gezeigt werden. Zentren, die ihre Teilnahme künftig reduzieren wollten, gaben gegenüber den oben genannten vergleichbare Problempunkte als Gründe an. Zu- 
Tab. 2. Beweggründe für eine Teilnahme an IIT

\begin{tabular}{ll}
\hline Aspekte & Mittelwert \\
\hline Verbesserung der Heilungschancen & 1,6 \\
Wissenschaftliches Interesse & 1,7 \\
Qualitätsmerkmal in der Außendarstellung & 1,8 \\
Anbindung an eine erfahrene Studiengruppe & 2,1 \\
Erfahrungsaustausch & 2,3 \\
Ethische Gründe & 2,4 \\
Beratung durch die jeweilige Studienzentrale & 2,5 \\
Zugang zu moderner Diagnostik & 2,6 \\
Verbesserung der Qualifikation des Prüfzentrums und gegebenenfalls Teilnahme an Pharmastudien & 2,8 \\
Fortbildungen bei Studientreffen & 3,0 \\
Erleichterung der Kostenerstattung durch Kostenträger & 3,3 \\
\hline
\end{tabular}

sätzlich verwiesen $41 \%$ der Zentren auf die Möglichkeit, Patienten auch analog zum Studienprotokoll ohne offizielle Meldung zu behandeln.

\section{Motivation und Gründe}

Für die Umfrageteilnehmer gab es eine Vielzahl an Aspekten, die sich auf ihre Motivation, an einer IIT teilzunehmen, auswirkten. Die Aspekte wurden auf einer Skala von 1-6 (sehr wichtiger bis überhaupt nicht wichtiger Aspekt) bewertet. Den höchsten Stellenwert (Mittelwert) hatten die Verbesserung der Heilungschancen $(1,6)$, das wissenschaftliche Interesse $(1,7)$ und die Bedeutung der Studienteilnahme als Qualitätsmerkmal in der Außendarstellung $(1,8)$. Andere Gründe waren: Anbindung an eine erfahrene Studiengruppe (2,1), Erfahrungsaustausch $(2,3)$, ethische Gründe $(2,4)$, Beratung durch die jeweilige Studienzentrale (2,5), Zugang zu moderner Diagnostik (2,6), Verbesserung der Qualifikation des Prüfzentrums und gegebenenfalls Teilnahme an Pharmastudien $(2,8)$, Fortbildungen bei Studientreffen $(3,0)$ sowie Erleichterung der Kostenerstattung durch Kostenträger $(3,3)$ (Tab. 2).

Bei Kliniken, die künftig ihre Teilnahme an IIT reduzieren wollen, waren die Entscheidungskriterien ähnlich verteilt. Als zusätzliche wichtige Punkte wurden die Häufigkeit der Indikation $(1,9)$ und der Dokumentationsaufwand $(2,0)$ angegeben. Die kostenfreie Bereitstellung der Medikation und die Altersgruppe wurden als weniger wichtig bewertet (3,5 bzw. $4,2)$.

\section{Unterstützungsbedarf teilnehmender Kliniken}

Die Umfrageteilnehmer wurden gebeten, Möglichkeiten zur Unterstützung bei der Studiendurchführung auf einer Skala (von 1 = sehr gutes Modell/Angebot bis $6=$ sehr schlechtes Modell/Angebot) zu bewerten. In absteigender Reihenfolge wurden folgende Bewertungen abgegeben (Mittelwert): zentrale Daten- und Dokumentebank für Qualifikationsunterlagen für Ethikkommissionen $(2,0)$, rückwirkende Zahlung von Fallgeldern (2,1), studienbezogene Diagnosis Related Groups (2,2), Bereitstellung von Unterlagen zum Qualitätsmanagement $(2,5)$, personelle Dokumentationsunterstützung
$(2,5)$, Unterstützung bei der Beantragung von neuen Untersuchungs- und Behandlungsmethoden $(2,5)$ und Konzept der integrierten Versorgung $(3,0)$.

\section{Diskussion}

An der Befragung haben $24 \%$ der angeschriebenen Praxen und $37 \%$ der Krankenhäuser (von Letzteren eher größere Häuser) teilgenommen. Die große Bedeutung von TOS wurde dabei an keiner Stelle infrage gestellt. Schwierigkeiten wurden eher in den konkreten Fragestellungen der Umfrage und dem damit verbundenen Arbeitsaufwand gesehen. Hier besteht insbesondere das Problem, dass in vielen Kliniken Zahlen zu neu diagnostizierten Patienten einzelner Entitäten nicht vorliegen. Für die Bewertung der Umfrage muss berücksichtigt werden, dass daher die Zahlen von den Umfrageteilnehmern geschätzt wurden.

Bei den teilnehmenden Zentren handelt es sich um hämatologisch-onkologische Spezialabteilungen oder Praxen mit vollständiger Ausstattung. Laboratoriumsdiagnostik, bildgebende Diagnostik, Intensivbehandlung und Notfallversorgung sind durchgehend gegeben. Viele Zentren setzen ein Qualitätsmanagement ein und arbeiten nach Standardanweisungen, sind zertifiziert oder planen eine Zertifizierung. Ethikkommissionen bewerten die Eignung eines Prüfzentrums für die Studienteilnahme auch aufgrund der oben genannten Angaben zur Ausstattung und Notfallversorgung für jede Studie neu. Die Ergebnisse dieser Umfrage deuten darauf hin, dass diese Prüfungen für die Teilnahme an einer multizentrischen IIT, die keine von der Standardversorgung abweichenden, diagnostischen oder apparativen Geräte oder medizinischen Kenntnisse erfordert, entfallen können.

Die Prüfzentren in der Hämatologie/Onkologie beteiligen sich zum Teil seit Jahrzehnten an IIT und besitzen darüber hinaus Erfahrung in der Durchführung von Studien der pharmazeutischen Industrie. Für viele hämatologisch-onkologische Erkrankungen gibt es keine Standardbehandlung. Es ist ein Qualitätsmerkmal der Versorgung, dass in diesen Entitäten die Rekrutierung in TOS den Standard darstellt und so 
Versorgung und Forschung verbunden werden. Die Umfrageergebnisse bestätigen den hohen Rekrutierungsanteil für akute Leukämien und Hodgkin-Lymphome. Auch für andere Erkrankungen werden hohe Rekrutierungsanteile erreicht. Die Schwankungsbreite ist zum einen darauf zurückzuführen, dass für einige Entitäten im Rahmen sukzessiver Therapieoptimierungen Standardbehandlungen für die Erstlinientherapie definiert werden konnten. Auf der anderen Seite können aber - auch aufgrund der Rahmenbedingungen - keine Studien angeboten werden oder der Aufwand der Studienteilnahme bei Erkrankungen mit besonders niedriger oder hoher Inzidenz wird als nicht leistbar eingeschätzt.

Zulassungsbehörden (Bundesinstitut für Arzneimittel und Medizinprodukte/Paul-Ehrlich-Institut (BfArM/PEI)) berichten in den Jahren seit der AMG-Novelle 2004 über einen gleich bleibenden Anteil (20\%) von nichtkommerziellen Studien an der Gesamtzahl beantragter Studien. Diese Zahlen erlauben jedoch keine Aussage dazu, wie sich die Anzahl von Studien mit hoher Fallzahl und die Anzahl teilnehmender Zentren entwickeln. Zudem liegen keine Zahlen zu den Indikationsgebieten vor und es kann nicht dargestellt werden, wie hoch der Anteil rein akademischer Studien ohne Industrieunterstützung ist. Demgegenüber berichten Ethikkommissionen über eine - teilweise starke - Reduktion der Anzahl von IIT durch verschiedene formelle Hürden [8]. Eine vergleichende europaweite Analyse in den Jahren 2003 und 2007 (vor bzw. nach der EU-Direktive) zeigt, dass die Anzahl der nichtkommerziellen Studien und auch die Anzahl der rekrutierenden Länder und Prüfzentren zurückgegangen ist [9]. Von der EU wird zudem für den Zeitraum 2007-2009 ein besorgniserregender Rückgang sowohl von Studien um 10\% als auch von eingeschlossenen Patienten in klinische Studien berichtet [10].

In den Kompetenznetzen im Bereich der Hämatologie und Onkologie werden ein Rückgang und eine deutliche Verzögerung großer TOS beobachtet. Demgegenüber besteht ein Trend zu kleinen Studien mit kurzer Laufzeit und finanzieller Unterstützung durch Unternehmen der pharmazeutischen Industrie. Diese Situation muss im Hinblick auf die Versorgungsqualität kritisch gewertet werden. Die Umfrageergebnisse zeigen, dass darüber hinaus künftig mit einer geringeren Teilnahme an IIT zu rechnen ist. Dies betrifft ein Drittel der Umfrageteilnehmer und signifikant stärker Praxen im Vergleich zu Kliniken. Aber auch Kliniken mit mittleren oder hohen Fallzahlen werden sich künftig weniger an Studien beteiligen. Diese Entwicklung wird die Möglichkeiten klinischer Forschung gerade bei seltenen Erkrankungen künftig einschränken.

Aus Sicht der Umfrageteilnehmer ist der Stellenwert von IIT insgesamt sehr hoch. Dies betrifft sowohl die ärztlichen und nichtärztlichen Studienmitarbeiter als auch die betroffenen Patienten. Nach der Einschätzung der Umfrageteilnehmer stehen $85 \%$ der Patienten einer Studienteilnahme positiv gegenüber. Besondere Vorteile einer Studienteilnahme von Patienten, gerade in TOS, bestehen in der umfassenden und detaillierten Aufklärung und letztlich in der Behandlung in einem Expertenverbund nach aktuellem wissenschaftlichen Standard. Patienten erleben ihre betreuenden Studienärzte insgesamt als einfühlsam und fühlen sich umfassender informiert [11].

Die Befragung im Hinblick auf die Motivation der Kliniken für eine Beteiligung an IIT zeigt, dass übergeordnete ethisch-wissenschaftliche Motive im Vordergrund stehen, z.B. reizvolle wissenschaftliche Fragestellungen oder die Chance auf Verbesserung der Heilungschancen. Aber auch andere Aspekte, die sich mit der Anbindung an Studiengruppen ergeben, wurden positiv bewertet. Dem gegenüber stehen jedoch relevante Nachteile, wie beispielsweise der - in Relation zur Fallzahl - sehr hohe interne Organisations- und Verwaltungsaufwand für die Studienbeteiligung bei personell nicht ausreichend ausgestatteten Prüfzentren. Dies führt zu Verzögerungen in der Studienaktivierung, zur Reduktion des Angebots am Zentrum und dadurch eventuell zum Rückgang der Rekrutierungszahlen. Problematisch ist insbesondere, dass die Beteiligung an klinischen Studien zwar gesundheitspolitisch gefordert wird, aber im Hinblick auf IIT kein adäquater finanzieller Ausgleich für den Mehraufwand der Kliniken und Praxen bezahlt wird.

In dieser Situation ist es notwendig, praktische Unterstützung anzubieten und Erleichterungen zu schaffen, die speziell auf multizentrisch angelegte TOS ausgerichtet sind, wie z.B. eine Alternative zum zeitaufwendigen Einsammeln von Qualifikationsprofilen an vielen Zentren. Die hierfür intensive Kommunikation beinhaltet mehrfaches Anfragen und Erinnern sowie eine Folgekommunikation, wenn die Unterlagen nicht aktuell oder vollständig sind. Bezüglich der Unterstützungsmöglichkeiten wurde in der Umfrage an erster Stelle die Bereitstellung einer zentralen Datenbank für Qualifikationsunterlagen genannt. Ein erster Prototyp «QualiPRO» wurde im Kompetenznetz Leukämien erarbeitet. Mit dieser Datenbank können die Abläufe durch Bereitstellung geeigneter Software-Werkzeuge und qualitätsgesicherter Prozesse für Erstellung, Sammlung, Nutzung und Bereitstellung von Qualifikationsunterlagen (z.B. Zentrumsqualifikation, Lebenslauf, Studienerfahrung) in einer internetbasierten Datenbank rationalisiert werden. Die Erweiterung des Prototyps unter Einbeziehung der Ethikkommissionen ist beantragt.

Vielfältige Bemühungen, auf nationaler und europäischer Ebene eine Änderung der gesetzlichen Rahmenbedingungen für IIT herbeizuführen, haben bisher zu keinem Erfolg geführt, obwohl die Bedeutung von IIT allgemein anerkannt wird. Dies betrifft sowohl die Novellierungen des deutschen AMG und der GCP-Verordnung als auch das geplante Amendment der entsprechenden EU-Direktive 2001/20/EC.

Akademische Studiengruppen sind im Vergleich zu Unternehmen der pharmazeutischen Industrie in den Möglichkeiten einer Lobbyarbeit sehr beschränkt. Es ist fraglich, ob es auch aufgrund der komplexen Interessenlage - gelingen wird, eine für IIT effektive Novellierung der gesetzlichen Bestim- 
mungen auf europäischer Ebene herbeizuführen. In Deutschland wurden zwar minimale Erleichterungen für nichtkommerzielle klinische Prüfungen in einem Papier des BfArM und PEI zusammengefasst [12] und die Bedeutung von IIT wird auch auf politischer Ebene wahrgenommen [13]. In Deutschland wurde im vergangenen Jahr der Entwurf eines «Zweiten Gesetzes zur Änderung arzneimittelrechtlicher und anderer Vorschriften» im Bundestag und Bundesrat diskutiert, das sich unter anderem auf das AMG und auf die GCPVerordnung auswirken soll [14]. Insgesamt sollen die mittlerweile verabschiedeten Änderungen dazu dienen, den verwaltungstechnischen Aufwand zu reduzieren. Dies beinhaltet neben anderen Änderungsvorschlägen -, dass nur noch Eignungsnachweise eines Prüfers und des Stellvertreters (statt wie bisher aller) bei der jeweiligen Ethikkommission vorgelegt werden sollen. Der Prüfer hat nun die Aufgabe, als «verantwortlicher Leiter der Prüfgruppe einer Prüfstelle, (...) für die Auswahl angemessener qualifizierter Mitglieder der Prüfgruppe (in der Regel Ärztinnen und Ärzte) zu sorgen, diese anzuleiten, zu informieren und zu überwachen und das Verfahren zu dokumentieren». Der Gesetzesentwurf wurde im Oktober 2012 verabschiedet; die praktische Umsetzung durch die Ethikkommissionen bleibt abzuwarten.

Auch daraus resultieren noch nicht umfassend genug die notwendigen praktischen Erleichterungen für Studiengruppen und Prüfzentren. Es bleibt weiterhin ein Spielraum offen, auch im Rahmen der aktuellen Gesetzeslage, weitere Erleichterungen zu schaffen, z.B. Gebührenreduktionen für TOS durch Ethikkommissionen und Bundesoberbehörden. Dieser sollte, auch im Hinblick auf die langwierigen Prozesse im Bezug auf Gesetzesänderungen, stärker genutzt werden.

\section{Fazit}

Es besteht eine Bedrohung der akademischen Forschungslandschaft durch gesetzliche Vorgaben. Trotz großer Akzeptanz der IIT und der Motivation der Zentren führt der erhöhte bürokratische Aufwand und die mangelnde finanzielle Unterstützung der Kliniken und Praxen zu einem Rückgang der Studienteilnahme, zu einer Abnahme der Rekrutierungszahlen und zu verlängerten Studienlaufzeiten. Gerade bei seltenen Entitäten ist auch eine studienanaloge Behandlung möglich, wobei der Aufwand einer Studienteilnahme entfällt. Dies hat negative Folgen für die Patienten, da weder Heilungschancen verbessert noch Daten zur Sicherheit erfasst werden, und für den Wissenschaftsstandort, da der hohe Stellenwert deutscher Studiengruppen im internationalen wissenschaftlichen Vergleich gefährdet ist. Bemühungen des BMBF, durch Förderprogramme TOS breit in der Versorgung zu aktivieren (z.B. Kompetenznetze), werden durch die gleichzeitig veränderten gesetzlichen Rahmenbedingungen konterkariert.

\section{Förderung}

Mit Unterstützung durch das BMBF (Fördernummer 01Gl9971/8) und die Deutsche José Carreras Leukämie-Stiftung (Fördernummer H06/04v).

\section{Disclosure Statement}

Die Autoren haben für diese Arbeit keine Interessenkonflikte offenzulegen.

\section{Literatur}

1 Goekbuget N, Hoelzer D: Bedeutung von Multicenterstudiengruppen für die klinische Forschung in der Hämatologie und Onkologie. Bundesgesundheitsblatt 2009;52:417-424

2 Elwyn G, Seagrove A, Thorne K, Cheung WY Ethics and research governance in a multicentre study: add 150 days to your study protocol. BMJ 2005;330:847.

3 Hackshaw A, Farrant H, Bulley S, Seckl MJ, Ledermann JA: Setting up non-commercial clinical trials takes too long in the UK: findings from a prospective study. J R Soc Med 2008;101:299-304.

4 Hearn J, Sullivan R: The impact of the 'Clinica Trials' directive on the cost and conduct of noncommercial cancer trials in the UK. Eur J Cancer 2007;43:8-13
5 Hemminki A, Kellokumpu-Lehtinen PL: Harmful impact of EU clinical trials directive. BMJ 2006; 332:501-502.

6 Tyndall A: Why do we need noncommercial, investigator-initiated clinical trials? Nat Clin Pract Rheumatol 2008;4:354-355.

7 Frewer LJ, Coles D, Champion K, Demotes-Mainard J, Goetbuget N, Ihrig K, Klingmann I, Kubiak C, Lejeune SA, McDonald F, Apperley J: Has the European Clinical Trials Directive been a success? BMJ 2010;340:c1862.

8 Schwarz S, Frölich L, Striebel J, Hennerici MG: Das Deutsche Arzneimittelgesetz: Ein Hindernis für nicht-kommerzielle klinische Studien? Dtsch Med Wochenschr 2007;19:108-112.
9 Klingmann I: Impact on clinical research of European legislation (ICREL). www.efgcp be/downloads/ icrel_docs/Final_report_ICREL $p d f$.

10 Führing S: (DG Research): At the Conference on Future of Independent Academic Clinical Research in Europe. www.eortc.org/newsletter-archive/futureindependent-academic-clinical-research-europe.

11 Gaul C, Schmidt T, Helm J, Hoyer H, Harting J: Motivation und Barrieren für die Teilnahme an klinischen Studien. Med Klin 2006;101:873-879.

12 Bundesinstitut für Arzneimittel und Medizinprodukte, Paul-Ehrlich-Institut, Bundesministerium für Gesundheit: Nicht-kommerzielle klinische Prüfungen. www.bfarm.de.

13 Deutscher Bundestag: BT-Drs. 17/1929; 2010.

14 Deutscher Bundestag: BT-Drs. 17/9341; 2012. 